\title{
NECESSARY AND SUFFICIENT CONDITIONS \\ FOR THE SOLVABILITY OF \\ A NONLINEAR TWO-POINT BOUNDARY VALUE PROBLEM
}

\author{
J. MAWHIN, J. R. WARD AND M. WILLEM
}

\begin{abstract}
The dual least action principle is used to prove a necessary and sufficient condition for the solvability of a Dirichlet problem of the form $u^{\prime \prime}+u+f(x, u)=0$. $u(0)=u(\pi)=0$ when $f(x, \cdot)$ is nondecreasing and $\int_{0}^{u} f\left(x, v^{\prime}\right) d v$ satisfies a suitable growth condition.
\end{abstract}

1. Introduction. Special cases of the Dirichlet problem

$$
\begin{aligned}
& u^{\prime \prime}(x)+u(x)+f(x, u(x))=0, \quad x \in I=[0, \pi] . \\
& u(0)=u(\pi)=0,
\end{aligned}
$$

where $f(x, \cdot)$ is nondecreasing for each fixed $x \in I$, have been recently considered by Fučik [6], Schechter, Shapiro and Snow [9] and Cesari and Kannan [4], who considered the existence of a solution of (1) when $f(x, u)=g(u)-h(x)$ with $h \in L^{2}(I), g$ continuous nondecreasing and

$$
|g(u)| \leqslant C_{1}+C_{2}|u|, \quad u \in \mathbf{R},
$$

for some $C_{1} \geqslant 0$ and $C_{2}>0$. In [6], the existence is obtained under the supplementary conditions that

$$
g(-u)=-g(u), \quad \lim _{n \rightarrow+\infty} g(u)=+\infty
$$

and $C_{2}<0.0962$, and this condition is improved in [9] to $C_{2}<0.24347$. Those supplementary conditions are partly weakened in [4] into

$$
\limsup _{|u| \rightarrow \infty} \frac{g(u)}{u}=C_{2}
$$

and

$$
C_{2}<0.443 \text {. }
$$

All those results are proved by a combination of the alternative method and Leray-Schauder degree arguments.

Received by the editors January 31, 1984.

1980 Mathematics Subject Classification. Primary 34B15, 49A40, 58E30.

Key words and phrases. Dirichlet problem, dual variational method, semilinear equations at resonance. jumping nonlinearities. 
That a condition of the form (2) and (5) cannot be completely avoided is shown by the elementary example (corresponding to $g(u)=3 u$ )

$$
\begin{aligned}
& u^{\prime \prime}(x)+4 u(x)=\sin 2 x, \quad x \in I, \\
& u(0)=u(\pi)=0,
\end{aligned}
$$

which has no solution, and that a condition of the form (3) or (4) cannot as well be completely avoided is shown by the elementary example (corresponding to $g(u)=0$ )

$$
\begin{aligned}
& u^{\prime \prime}(x)+u(x)=\sin x, \quad x \in I, \\
& u(0)=u(\pi)=0,
\end{aligned}
$$

which has no solution as well. The aim of this paper is to use the dual least action principle of Clarke and Ekeland [5] (see [8, 10] for details and references) to improve substantially the above quoted results for problem (1) in the following way. We first replace (2)-(5) by a condition on $F(x, u)=\int_{0}^{u} f(s, v) d s$ explicity stated below and which is implied, in the special case of $f(x, u)=g(u)-h(x)$, by condition (2) with the sole restriction

$$
0<C_{2}<3
$$

We notice that (8) is optimal with respect to example (6). Under this restriction on $F$ we then state a necessary and sufficient condition on $f$ under which the problem (1) has at least one solution. Needless to say, this necessary and sufficient condition contains as special cases the conditions (3) or (4), as it can be checked in a very elementary way, and, in the case of $g=0$, it reduces exactly to the condition (violated in example (7))

$$
\int_{0}^{\pi} h(x) \sin x d x=0
$$

of the Fredholm alternative for the problem

$$
u^{\prime \prime}+u=h(x), \quad u(0)=u(\pi)=0 .
$$

Notice also that when $f(x, \cdot)$ is nonincreasing for each fixed $x \in I$, then a simple upper and lower solution argument, like in Kazdan and Warner [7], shows that our condition on $f$ without any growth restriction on $F$ is necessary and sufficient for the solvability of (1).

We now state explicitly the theorem proved in this paper. Let $v^{+}=\max (v, 0)$, $v^{-}=\max (-v, 0)$ and let us say that $f: I \times \mathbf{R} \rightarrow \mathbf{R}$ satisfies the Carathéodory conditions for $L^{2}(I)$ when $f(x, \cdot)$ is continuous for a.e. $x \in I, f(\cdot, u)$ measurable for each $u \in \mathbf{R}$, and $|f(x, u)| \leqslant h_{R}(x)$ whenever $|u| \leqslant R$ with $h_{R} \in L^{2}(I)$.

THEOREM 1. Assume that $f: I \times \mathbf{R} \rightarrow \mathbf{R}$ satisfies the Carathéodory conditions for $L^{2}(I)$ and that $f(x, \cdot)$ is nondecreasing for a.e. $x \in I$. Assume, moreover, that there exists $\alpha_{+} \in L^{\infty}(I)$ and $\alpha_{-} \in L^{\infty}(I)$ such that:

(i) $\inf _{I}$ ess $\alpha_{+}>0$, inf ${ }_{I}$ ess $\alpha_{-}>0$.

(ii) $\alpha_{+}(x) \leqslant 3$ and $\alpha_{-}(x) \leqslant 3$ for a.e. $x \in I$.

(iii) $\int_{0}^{\pi}\left\{\left[3-\alpha_{+}(x)\right]\left(\sin ^{+} 2 x\right)^{2}+\left[3-\alpha_{-}(x)\right]\left(\sin ^{-} 2 x\right)^{2}\right\} d x>0$. 
(iv) For each $\varepsilon>0$ there is a $\beta_{-}\left(\right.$resp. $\left.\beta_{+}\right)$in $L^{1}(I)$ such that for a.e. $x \in I$ and all $u<0$ (resp. $u>0$ ) we have

$$
2 u^{-2} F(x, u) \leqslant \alpha_{-}(x)+u^{-2} \beta_{-}(x)+\varepsilon \quad\left(\operatorname{resp} . \alpha_{+}(x)+u^{-2} \beta_{+}(x)+\varepsilon\right) .
$$

Then problem (1) has at least one solution if and only if there exists $a \in \mathbf{R}$ such that

$$
\bar{f}(a) \equiv \int_{0}^{\pi} f(x, a \sin x) \sin x d x=0 .
$$

Notice that (9) is equivalent to the existence of a critical point for the function $\bar{F}$ : $\mathbf{R} \rightarrow \mathbf{R}$ defined by

$$
\bar{F}(a)=\int_{0}^{\pi} F(x, a \sin d x) d x
$$

so that a sufficient condition for (9) to hold will be that either $\bar{F}(a) \rightarrow+\infty$ or $\bar{F}(a) \rightarrow-\infty$ whenever $|a| \rightarrow \infty$. Conditions of this type were first introduced by Ahmad, Lazer and Paul [2].

The proof of Theorem 1 will be given in $\S 3$ and some preliminary lemmas are stated and proved in $\S 2$.

When (2) is satisfied, we have

$$
F(x, u) \leqslant C_{1}|u|+\frac{1}{2} C_{2}|u|^{2}+|h(x)||u|
$$

and, hence, for each $\varepsilon>0$

$$
F(x, u) \leqslant \frac{1}{2}\left(C_{2}+\varepsilon\right) u^{2}+\frac{1}{2 \varepsilon}\left[C_{1}+|h(x)|\right]^{2} .
$$

Therefore, if we take $\alpha_{+}=\alpha_{-}=C_{2}$, conditions (i)-(iv) reduce to (8). But, by a result of Brézis-Nirenberg, condition (10) implies for $f$ a growth restriction of the form

$$
|f(x, u)| \leqslant C_{3}(x)+2\left(C_{2}+\varepsilon\right)|u|,
$$

and the constant $2\left(C_{2}+\varepsilon\right)$ is indeed optimal (see e.g. [8 and 10] where details and reference about the used variational techniques can be found).

We conclude this Introduction by exhibiting an example which deals with jumping nonlinearities (i.e. when $\left.\alpha_{-} \neq \alpha_{+}\right)$. If we take $f(x, u)=c u^{+}-h(x)$ for some $c \in \mathbf{R}$, then it is easily shown that

$$
\limsup _{u \rightarrow-\infty} 2 F(x, u) / u^{2}=0, \quad \limsup _{u \rightarrow+\infty} 2 F(x, u) / u^{2}=c
$$

in the sense of (iv) and, by taking $\alpha_{+}(x)=c$ and $\alpha_{-}(x)$ equal to any constant in ]0,3[ we see that conditions (i)-(iii) are satisfied if

$$
0<c \leqslant 3 \text {. }
$$

Moreover,

$$
\bar{f}(a)= \begin{cases}-\int_{0}^{\pi} h(x) \sin x d x & \text { if } a \leqslant 0 \\ 2 c a-\int_{0}^{\pi} h(x) \sin x d x & \text { if } a>0\end{cases}
$$


which shows that the problem

$$
\begin{aligned}
& u^{\prime \prime}(x)+u(x)+c u^{+}(x)=h(x), \quad x \in I, \\
& u(0)=u(\pi)=0
\end{aligned}
$$

has a solution, when (12) holds, if and only if

$$
\int_{0}^{\pi} h(x) \sin x d x \geqslant 0
$$

This complements results of Aguinaldo and Schmitt [1] and Castro [3] dealing with problems of the form

$$
\begin{aligned}
& u^{\prime \prime}(x)+u(x)-c u^{-}(x)=h(x), \quad x \in I, \\
& u(0)=u(\pi)=0
\end{aligned}
$$

with $c>0$, i.e. with jumping nonlinearities with graph below that of the line $v=u$, in contrast with our situation which covers jumping nonlinearities with graph between the lines $v=u$ and $v=4 u$.

2. Preliminary lemmas. We state and prove simple preliminary results required in the proof of Theorem 1 . For every $h \in L^{2}(I)$, such that

$$
\int_{0}^{\pi} h(x) \sin x d x=0
$$

let us denote by $K h$ the unique solution of the problem

$$
u^{\prime \prime}(x)+u(x)=h(x), \quad u(0)=u(\pi)=0,
$$

such that

$$
\int_{0}^{\pi} u(x) \sin x d x=0
$$

Moreover, for each $v \in L^{2}(I)$, let us write

$$
v(x)=\bar{v}(x)+\tilde{v}(x),
$$

where

$$
\bar{v}(x)=\frac{2}{\pi}\left(\int_{0}^{\pi} v(s) \sin s d s\right) \sin x \text { and } \int_{0}^{\pi} \tilde{v}(s) \sin s d s=0,
$$

so that, with obvious notation, $L^{2}(I)=\bar{L}^{2}(I) \oplus \tilde{L}^{2}(I)$. Using Fourier series and Parseval equality, it is easy to show that the inequality

$$
\int_{I} w(x)(K w)(x) d x \geqslant-\frac{1}{3} \int_{I} w^{2}(x) d x
$$

holds for every $w \in \tilde{L}^{2}(I)$ with equality if and only if $w$ has the form $w(x)=B \sin 2 x$ for some $B \in \mathbf{R}$.

Lemma 1. Let $\alpha_{+} \in L^{\infty}(I)$ and $\alpha_{-} \in L^{\infty}(I)$ be such that the assumptions (i)-(iii) of Theorem 1 are satisfied. Then there exists $\delta=\delta\left(\alpha_{+}, \alpha_{-}\right)>0$ such that for each 
$w \in \tilde{L}^{2}(I)$ one has

$$
\begin{aligned}
\gamma(w) & \equiv \frac{1}{2} \int_{I}\left\{w(x)(K w)(x)+\frac{\left[w^{+}(x)\right]^{2}}{\alpha_{+}(x)}+\frac{\left[w^{-}(x)\right]^{2}}{\alpha_{-}(x)}\right\} d x \\
& \geqslant \delta \int_{I} w^{2}(x) d x .
\end{aligned}
$$

Proof. We first show that $\gamma(w)=0$ if and only if $w=0$. Clearly, $\gamma(0)=0$. Now, if $\gamma(w)=0$, then by (13)

$$
0=\gamma(w) \geqslant \frac{1}{2} \int_{l}\left\{\left(\frac{1}{\alpha_{+}(x)}-\frac{1}{3}\right)\left[w^{+}(x)\right]^{2}+\left(\frac{1}{\alpha_{-}(x)}-\frac{1}{3}\right)\left[w^{-}(x)\right]^{2}\right\} d x \geqslant 0
$$

and by assumption (ii) and (13)

$$
0=\gamma(w) \geqslant \frac{1}{2} \int_{I}\left[w(x)(K w)(x)+\frac{1}{3} w^{2}(x)\right] d x \geqslant 0
$$

so that, necessarily, $w(x)=B \sin 2 x$. Introduced in (14), this gives

$$
\frac{B^{2}}{2} \int_{I}\left[\left(\frac{1}{\alpha_{+}(x)}-\frac{1}{3}\right)\left(\sin ^{+} 2 x\right)^{2}+\left(\frac{1}{\alpha_{-}(x)}-\frac{1}{3}\right)\left(\sin ^{-} 2 x\right)^{2}\right] d x=0,
$$

and hence $B=0$ by condition (iii). Now assume that the conclusion of the lemma is false. There will be a sequence $\left(w_{n}\right)$ in $\tilde{L}^{2}(I)$ and a $w \in \tilde{L}^{2}(I)$ such that

$$
\left\|w_{n}\right\|_{L^{2}}=1, \quad w_{n}^{ \pm} \rightarrow w^{ \pm}, \quad K w_{n} \rightarrow K w, \quad \gamma\left(w_{n}\right) \rightarrow 0
$$

when $n \rightarrow \infty$. Consequently,

$$
\int_{I}\left\{\frac{\left[w_{n}^{+}(x)\right]^{2}}{\alpha_{+}(x)}+\frac{\left[w_{n}^{-}(x)\right]^{2}}{\alpha_{-}(x)}\right\} d x \rightarrow-\int_{I} w(x)(K w(x)) d x
$$

and, hence, by weak lower semicontinuity of a continuous convex functional,

$$
\int_{I}\left\{\frac{\left[w^{+}(x)\right]^{2}}{\alpha_{+}(x)}+\frac{\left[w^{-}(x)\right]^{2}}{\alpha_{-}(x)}\right\} d x \leqslant-\int_{I} w(x)(K w(x)) d x,
$$

which gives $\gamma(w) \leqslant 0$ and hence $w=0$. But then, as

$$
\gamma\left(w_{n}\right) \geqslant \frac{1}{2} \int_{I} w_{n}(x)\left(K w_{n}(x)\right)(x) d x+\frac{1}{6} \geqslant 0,
$$

we get a contradiction letting $n \rightarrow \infty$.

The following lemma will provide the proof of the necessity of (9).

LEMma 2. Assume that the function $f: I \times \mathbf{R} \rightarrow \mathbf{R}$ satisfies the Carathéodory conditions for $L^{z}(I)$ and that $f(x, \cdot)$ is nondecreasing for a.e. $x \in I$. If (1) has a solution, then there exists $a \in \mathbf{R}$ such that (9) holds.

Proof. If (1) has a solution $u$, then by the Fredholm alternative

$$
\int_{0}^{\pi} f(x, u(x)) \sin x d x=0
$$


It follows from elementary arguments that for all $x \in I$

$$
\left|\frac{u(x)}{\sin x}\right| \leqslant \frac{\pi}{2} \max _{s \in I}\left|u^{\prime}(s)\right| \text {. }
$$

Therefore, using (15) and the monotonicity of $f(x, \cdot)$, we obtain, if $R=$ $\max _{s \in I}\left|u^{\prime}(s)\right|$,

$$
\bar{f}\left(-\frac{\pi}{2} R\right) \leqslant 0 \leqslant \bar{f}\left(\frac{\pi}{2} R\right)
$$

and the result follows from the intermediate value theorem.

3. Proof of Theorem 1. The necessity follows directly from Lemma 2 . We divide the sufficiency proof into two parts.

(a) Existence of a solution for a perturbed problem. Let $\varepsilon>0, f_{\varepsilon}(x, u)=f(x, u)+$ $\varepsilon u, F_{\varepsilon}(x, u)=\int_{0}^{u} f_{\varepsilon}(x, v) d v$ and let $\psi_{\varepsilon}$ be defined on $\tilde{L}^{2}(I)$ by

$$
\psi_{\varepsilon}(v)=\int_{I}\left[\frac{1}{2} v(x)(K v)(x)+F_{\varepsilon}^{*}(x, v(x))\right],
$$

where $F_{\varepsilon}^{*}(x, \cdot)$ denotes the Legendre transform of $F_{\varepsilon}(x, \cdot)$ implicitly defined by

$$
F_{\varepsilon}^{*}(x, v)=v u-F_{\varepsilon}(x, u), \quad v=f_{\varepsilon}(x, u)=\left(\partial F_{\varepsilon} / \partial u\right)(x, u),
$$

so that, alternatively,

$$
F_{\varepsilon}^{*}(x, v)=\sup _{x \in \mathbf{R}}\left[v w-F_{\varepsilon}(x, w)\right] .
$$

Let $\eta>0$ be such that

$$
\frac{1}{\alpha_{+}(x)+2 \eta} \geqslant\left[\frac{1}{\alpha_{+}(x)}\right]-\delta, \quad \frac{1}{\alpha_{-}(x)+2 \eta} \geqslant\left[\frac{1}{\alpha_{-}(x)}\right]-\delta,
$$

where $\delta=\delta\left(\alpha_{+}, \alpha_{-}\right)$is given by Lemma 1. By assumption (iv) there exists $\beta \in L^{2}(I)$ such that

$$
F(x, u) \leqslant\left(\alpha_{+}(x)+\eta\right)\left[\left(u^{+}\right)^{2} / 2\right]+\left(\alpha_{-}(x)+\eta\right)\left[\left(u^{-}\right)^{2} / 2\right]+\beta(x)
$$

for a.e. $x \in I$ and all $u \in \mathbf{R}$. Consequently, by (18) and (20), we get

$$
F_{\varepsilon}^{*}(x, v) \geqslant \frac{\left(v^{+}\right)^{2}}{2\left(\alpha_{+}(x)+\eta+\varepsilon\right)}+\frac{\left(v^{-}\right)^{2}}{2\left(\alpha_{-}(x)+\eta+\varepsilon\right)}-\beta(x)
$$

and therefore, if $0<\varepsilon \leqslant \eta$, we have by (19) and Lemma 1 ,

$$
\begin{aligned}
\psi_{\varepsilon}(v) & \geqslant \int_{I}\left\{\frac{1}{2} v(x)(K v)(x)+\frac{\left[v^{+}(x)\right]^{2}}{2 \alpha_{+}(x)}+\frac{\left[v^{-}(x)\right]^{2}}{2 \alpha_{-}(x)}-\frac{\delta}{2} v^{2}(x)-\beta(x)\right\} d x \\
\geqslant & \frac{\delta}{2}\|v\|_{L^{2}}^{2}-\|\beta\|_{L^{1}} .
\end{aligned}
$$

Thus $\psi_{\varepsilon}$ is coercive and is weakly lower semicontinuous as the sum of a weakly continuous and a continuous convex function. Consequently, $\psi_{\varepsilon}$ has a minimum $v_{\varepsilon}$ on $\tilde{L}^{2}(I)$ which satisfies the Euler equation

$$
K v_{\varepsilon}(x)+(\partial / \partial v) F_{\varepsilon}^{*}\left(x, v_{\varepsilon}(x)\right)=\bar{u}_{\varepsilon}(x), \quad x \in I,
$$


for some $\bar{u}_{\varepsilon} \in \bar{L}^{2}(I)$. Letting $u_{\varepsilon}=\bar{u}_{\varepsilon}-K v_{\varepsilon}$, we get

$$
u_{\varepsilon}(x)=(\partial / \partial v) F_{\varepsilon}^{*}\left(x, v_{\varepsilon}(x)\right), \quad x \in I .
$$

Consequently, by (17)

$$
v_{\varepsilon}(x)=(\partial / \partial u) F_{\varepsilon}\left(x, u_{\varepsilon}(x)\right), \quad x \in I,
$$

i.e. by definition of $K$

$$
\begin{aligned}
& u_{\varepsilon}^{\prime \prime}(x)+u_{\varepsilon}(x)+f_{\varepsilon}\left(x, u_{\varepsilon}(x)\right)=0, \quad x \in I, \\
& u_{\varepsilon}(0)=u_{\varepsilon}(\pi)=0 .
\end{aligned}
$$

(b) A posteriori estimates on $u_{\varepsilon}$ and limit for $\varepsilon \rightarrow 0$. By assumption (9) the function $v$, defined by

$$
v(x)=f(x, a \sin x),
$$

belongs to $\tilde{L}^{2}(I)$, and hence,

$$
\psi_{\varepsilon}\left(v_{\varepsilon}\right) \leqslant \psi_{\varepsilon}(v) \leqslant \int_{I}\left[\frac{1}{2} v(x)(K v)(x)+F^{*}(x, v(x))\right] d x,
$$

where, for a.e. $x \in I$,

$$
F^{*}(x, z)=\sup _{l \in \mathbf{R}}[z y-F(x, y)]
$$

is the Fenchel transform $F^{*}(x, \cdot)$ of the convex function $F(x, \cdot)$. Now (23) tells that $a \sin x$ is a critical point of the concave function $y \mapsto v(x) y-F(x, y)$ and hence an absolute maximum, so that

$$
F^{*}(x, v(x))=v(x) a \sin x-F(x, a \sin x) .
$$

From (21), (24) and (25) we deduce that

$$
\frac{\delta}{2} \int_{I} v_{\varepsilon}^{2}(x) d x \leqslant C(a)+\|\beta\|_{L^{1}}
$$

and hence,

$$
\left\|u_{\varepsilon}^{\prime \prime}+u_{\varepsilon}\right\|_{L^{2}} \leqslant C_{1}(a, \beta, \delta)
$$

for all $0<\varepsilon \leqslant \eta$. From

$$
\left\|u_{\varepsilon}^{\prime \prime}+u_{\varepsilon}\right\|_{L^{2}}=\left\|\tilde{u}_{\varepsilon}^{\prime \prime}+\tilde{u}_{\varepsilon}\right\|_{L^{2}} \geqslant 3\left\|\tilde{u}_{\varepsilon}\right\|_{L^{2}},
$$

we easily see that

$$
\left\|\tilde{u}_{\varepsilon}\right\|_{L^{2}} \leqslant C_{2}(a, \beta, \delta), \quad\left\|\tilde{u}_{\varepsilon}^{\prime \prime}\right\|_{L^{2}} \leqslant C_{3}(a, \beta, \delta)
$$

and hence,

$$
\left\|\tilde{u}_{\varepsilon}\right\|_{C^{1}} \leqslant C_{4}(a, \beta, \delta) .
$$

On the other hand, it follows from (22) that if $\bar{u}_{\varepsilon}(x)=a_{\varepsilon} \sin x$, then

$$
\varepsilon a_{\varepsilon}+\frac{2}{\pi} \int_{0}^{\pi} f\left(x, u_{\varepsilon}(x)\right) \sin x d x=0 .
$$

By (9), the monotonicity of $f(x, \cdot)$, inequality (16), and (26), such an equality is impossible if

$$
a_{\varepsilon}>|a|+(\pi / 2) C_{4}(a, \beta, \delta) \text { or } a_{\varepsilon}<-|a|-(\pi / 2) C_{4}(a, \beta, \delta)
$$


because, say, in the first case we should have on $[0, \pi]$

$$
\begin{aligned}
u_{\varepsilon}(x) & =a_{\varepsilon} \sin x+\tilde{u}_{\varepsilon}(x) \\
& \geqslant a \sin x+(\pi / 2) C_{4}(a, \beta, \delta) \sin x-(\pi / 2) C_{4}(a, \beta, \delta) \sin x \\
& =a \sin x
\end{aligned}
$$

and hence, the left-hand member of (27) should be positive. Consequently,

$$
\left|a_{\varepsilon}\right| \leqslant|a|+(\pi / 2) C_{4}(a, \beta, \delta)
$$

and then

$$
\left\|u_{\varepsilon}\right\|_{C^{1}} \leqslant C_{5}(a, \beta, \delta), \quad\left\|u_{\varepsilon}^{\prime \prime}\right\|_{L^{2}} \leqslant C_{6}(a, \beta, \delta) .
$$

Those conditions and Ascoli and Arzela's theorem imply the existence of $u \in C^{1}(I)$ and of a sequence $\left(\varepsilon_{n}\right)$ in $\left.] 0, \eta\right]$ such that $\varepsilon_{n} \rightarrow 0$ and $u_{\varepsilon_{n}} \rightarrow u$ in $C^{1}(I)$ when $n \rightarrow \infty$. Consequently, $u(0)=u(\pi)=0$ and, for each $n \in N$ and each $x \in I$,

$$
u_{\varepsilon_{n}}^{\prime}(x)-u_{\varepsilon_{n}}^{\prime}(0)+\int_{0}^{x}\left[u_{\varepsilon_{n}}(s)+f_{\varepsilon_{n}}\left(s, u_{\varepsilon_{n}}(s)\right)\right] d s=0 .
$$

Letting $n \rightarrow \infty$, we get

$$
u^{\prime}(x)-u^{\prime}(0)+\int_{0}^{x}[u(s)+f(s, u(s))] d s=0,
$$

i.e.

$$
u^{\prime \prime}(x)+u(x)+f(x, u(x))=0, \quad x \in I,
$$

and the proof is complete.

\section{REFERENCES}

1. L. Aguinaldo and K. Schmitt, On the boundary value problem $u^{\prime \prime}+u=\alpha u^{-}+p(t), u(0)=0=$ $u(\pi)$. Proc. Amer. Math. Soc. 68 (1978), 64-68.

2. S. Ahmad, A. C. Lazer and J. L. Paul, Elementary critical point theory and perturbations of elliptic boundary value problems at resonance, Indiana Univ. Math. J. 25 (1976), 933-944.

3. A. Castro, A two-point boundary value problem with jumping nonlinearities, Proc. Amer. Math. Soc. 79 (1980), 207-211.

4. L. Cesari and R. Kannan, Existence of solutions of a nonlinear differential equations, Proc. Amer. Math. Soc. 88 (1983), 605-613.

5. F. Clarke and I. Ekeland, Hamiltonian trajectories with prescribed minimal periods, Comm. Pure Appl. Math. 33 (1980), 103-116.

6. S. Fučik, Surjectivity of operators in solving linear noninvertible part and nonlinear compact perturhation, Funkcial. Ekvac. 17 (1974), 73-83.

7. J. L. Kazdan and F. Warner, Remarks on some quasilinear elliptic equations, Comm. Pure Appl. Math. 28 (1975), 567-597.

8. J. Mawhin, Compacité, monotonie et convexité dans l'étude de problèmes aux limites semi-linéaires, Sém. Anal. Moderne, No. 19 (Université de Sherbrooke, Québec, 1981).

9. M. Schechter, J. Shapiro and M. Snow, Solutions of the nonlinear problem $A u=N(u)$ in a Banach space, Trans. Amer. Math. Soc. 241 (1978), 69-78.

10. M. Willem, Lectures on critical point theory, Trabalho Mat., No. 199, Fundaçao Univ. Brasilia, 1983.

Université de Louvain, Institut Mathematique, B - 1348 Louvain - La - Neuve, Belgium

Department of Mathematics, University of Alabama, University, Alabama 35486

Université de Louvain, Institut Mathématique, B - 1348 Louvain - La - Neuve, Belgium 